\title{
Design and fabrication of gas cell targets for laboratory astrophysics experiments on the Orion high-power laser facility
}

\author{
C. Spindloe ${ }^{1}$, D. Wyatt ${ }^{1}$, S. Astbury ${ }^{1}$, G. F. Swadling ${ }^{2,5}$, T. Clayson ${ }^{2}$, C. Stehlé ${ }^{4}$, J. M. Foster ${ }^{3}$, \\ E. Gumbrell ${ }^{3,5}$, R. Charles ${ }^{3}$, C. N. Danson ${ }^{2,3}$, P. Brummitt ${ }^{1}$, and F. Suzuki-Vidal ${ }^{2}$ \\ ${ }^{1}$ Science and Technology Facilities Council, Harwell Science and Innovation Campus, Didcot, OX11 OQX, UK \\ ${ }^{2}$ Blackett Laboratory, Imperial College, London, SW7 2BW, UK \\ ${ }^{3} A W E$ plc, Aldermaston, Reading RG7 4PR, UK \\ ${ }^{4}$ LERMA, Sorbonne Universités, UPMC Univ. Paris 06, Observatoire de Paris, PSL Research University, CNRS, F-75252, Paris, France \\ ${ }^{5}$ Current address: Lawrence Livermore National Laboratory, California 94550, USA \\ (Received 21 February 2017; revised 19 April 2017; accepted 11 May 2017)
}

\begin{abstract}
This paper describes the design and fabrication of a range of 'gas cell' microtargets produced by the Target Fabrication Group in the Central Laser Facility (CLF) for academic access experiments on the Orion laser facility at the Atomic Weapons Establishment (AWE). The experiments were carried out by an academic consortium led by Imperial College London. The underlying target methodology was an evolution of a range of targets used for experiments on radiative shocks and involved the fabrication of a precision machined cell containing a number of apertures for interaction foils or diagnostic windows. The interior of the cell was gas-filled before laser irradiation. This paper details the assembly processes, thin film requirements and micro-machining processes needed to produce the targets. Also described is the implementation of a gas-fill system to produce targets that are filled to a pressure of $0.1-1$ bar. The paper discusses the challenges that are posed by such a target.
\end{abstract}

Keywords: astrophysics; microtargets

\section{Introduction}

As part of its commitment to the UK academic plasma physics community the Central Laser Facility (CLF) supports academic access to the Orion laser facility at the Atomic Weapons Establishment (AWE) Aldermaston ${ }^{[1]}$, which constitutes up to $15 \%$ of the Orion experimental programme through the supply of target components and subsistence provision. This target fabrication support is in addition to the experimental support provided at the Rutherford Appleton Laboratory (RAL) for the high-power laser experiments carried out for the CLF programme. Over the past 40 years the CLF Target Fabrication Group has developed extensive skills in a range of capabilities that are directly applicable to target manufacture and has dedicated laboratories for thin film coating, micro-assembly, metrology, wafer based target manufacture, laser micro-

Correspondence to: C. Spindloe.

Email: christopher.spindloe@stfc.ac.uk machining and precision micro-machining. In addition, the group runs a number of development programmes to increase the level of capability for UK user groups and to drive forward target technology. One such development that has been delivered is the implementation of a gas-filled microtarget capability. This was developed in response to an AWE academic access experiment ${ }^{[2,3]}$ carried out by a consortium led by Imperial College London and drawing on knowledge from AWE, Imperial College London, Observatoire de Paris and RAL, a capability was introduced and then transferred to RAL for the user community access for CLF experiments.

\section{Orion academic access experiment}

The requirement was to develop an experimental platform (target assembly) to produce and study counter-streaming radiative shocks relevant to astrophysics. This is a further 


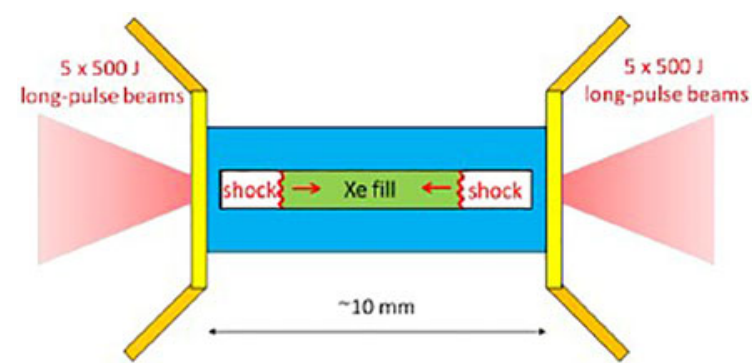

Figure 1. Experimental layout for the Orion gas cell campaign.

development of previous designs to enable the study of the more complex phenomena and instabilities that can arise in such a system ${ }^{[4]}$. Since 2000 there have been a number of designs for gas targets used in this field that have either had the features of tubes down which the plasma flow propagates or cells with windows ${ }^{[5-7]}$. The target design was optimized to allow for the shocks to propagate without interacting with the cell walls and for optical and X-ray diagnostics to be able to image the shocks as close as possible to their point of origin. The target cell was required to be filled with either argon, neon, krypton or xenon to pressures ranging from 0.1 to 1 bar with control of the internal gas pressure from shot to shot. The ends of the gas cell were sealed with solid ablators made from plastic disks; these are driven by the lasers and act as pistons in the gas cell to drive shocks. Other key target features were brominated plastic layers to prevent preheat of the gas, copper cones to shield the interaction point of the lasers from the diagnostics and a number of alignment features.

\section{Target design}

\subsection{Overall design}

The proposed experimental layout is shown in Figure 1 with a side, rectangular window for X-ray backlighter (XRBL) imaging of the counter-propagating shocks shown. Up to five of the Orion long-pulse beams can be used on each side of the target.

Through several iterations, a more complex cell was designed with an octagonal shape and two large $(5 \mathrm{~mm}$, subsequently changed to $3 \mathrm{~mm}$ ) open apertures at each end for the plastic ablators. The gas cell was machined from a single piece of aluminium using a computer numerical control (CNC) micro-mill to provide a joint-free component; this design is shown in Figure 2(b). The open apertures on each end had a stepped profile to allow the assembly of the shielding cones on the outside. These cones were pressed from $100 \pm 10 \mu \mathrm{m}$ thick copper sheet and these can be seen in Figure 2(a).

Two different sets of windows were used for both X-ray imaging and optical interferometry. The chosen material for

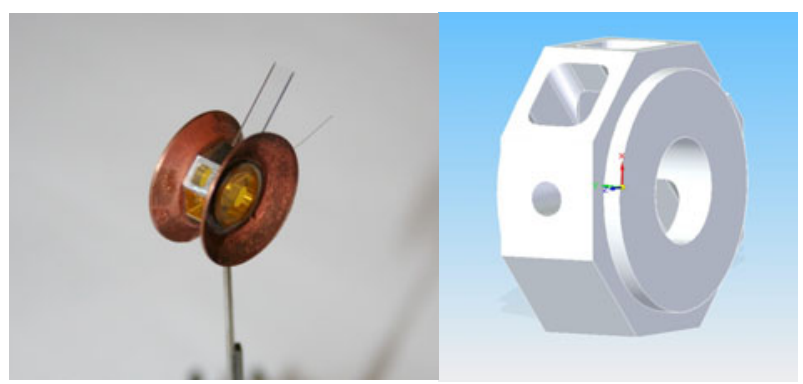

Figure 2. (a) Fully assembled target for Orion gas cell campaign and (b) the base gas cell design.

the X-ray windows was a polyimide plastic, laser-cut and adhered to the aperture on opposite sides. The thickness of the plastic was defined as $25 \pm 5 \mu \mathrm{m}$ (total X-ray path of $50 \mu \mathrm{m}$ through both windows) allowing a suitable transmission of X-rays (6.7 keV iron He-alpha generated from a $5 \mu \mathrm{m}$ iron foil) for the diagnostics. Optical windows were specified at $250 \mu \mathrm{m}$ thick and were anti-reflection coated to match the optical probe wavelength of $532 \mathrm{~nm}$. In addition to the two optical windows, a compensating glass block of total $500 \mu \mathrm{m}$ thickness was designed to be added to the top of the cell with the same anti-reflection coating. This allowed matching of path lengths for optical interferometry through the cell; however, this was not experimentally used.

The pusher design consisted of a $25 \pm 5 \mu \mathrm{m}$ polypropylene support window onto the inside of which was adhered a $50 \mu \mathrm{m}$ thick brominated plastic (CHBr) disk (details in Section 3.2). Finally, a thin gas-fill tube that also acts as the target support was added to the bottom of the cell and this was mounted to a standard Orion target mount for positioning in a Ten-Inch Manipulator (TIM) based target holder installed in the Orion chamber. The final prototype target assembly with the compensating glass is shown in Figure 2 with a fiducial wire for alignment visible.

\subsection{Brominated plastic disks}

The brominated plastic disks were produced using hotpressing techniques as a foil of the required bromine content, and thickness was not commercially available. Previous academic access experiments ${ }^{[8]}$ have used CHBr disks developed using hot-pressing a brominated plastic bromo-4polystyrene, which although pressed under temperature and pressure can form a foil with a granular structure, which would be unsuitable for the experiment as it would seed instabilities in the plasma flow.

The use of a different form of $\mathrm{CHBr}$ (poly-4bromostyrene) allowed the production of a clear disk with a reduction in the granularity in the bulk and at the surface. Characterization of the bromine content confirmed a $31.10 \mathrm{wt} \% \mathrm{Br}$ composition by mass ${ }^{[9]}$. An image of the improved disk is shown in Figure 3. When pressed the 


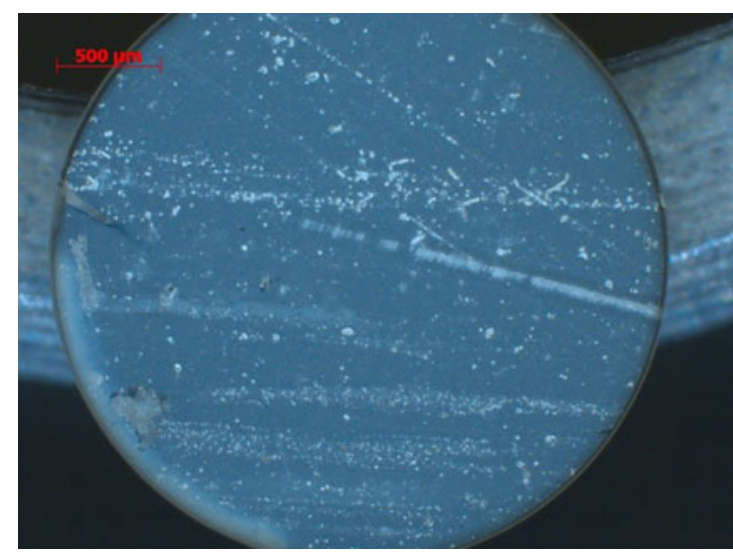

Figure 3. A brominated plastic disk before adhering to the polypropylene support on the gas cell.

thickness of the material varies across the disk and so using a touch probe sections that were $50 \pm 5 \mu \mathrm{m}$ were selected for the experiment. The brominated disk was adhered to a polypropylene window of $25 \mu \mathrm{m}$ thickness that spanned the full diameter of the end entrance hole of the cell of $5 \mathrm{~mm}$. This was subsequently reduced to $3 \mathrm{~mm}$ to reduce the bowing of the windows due to pressure differentials when in the chamber and in filling. The brominated plastic disk was laser-cut to a diameter of $2 \mathrm{~mm}$ as it was brittle and adhering to the full diameter generally led to cracking of the disk. The brominated disk was adhered to the polypropylene disk with a 2-part araldite and transmitted light illumination showed up any voids in the glue layer. Targets with voids were not used. Touch probe measurements of the assembled part indicated glue layers of 3-5 $\mu \mathrm{m}$.

\subsection{Adhesive selection}

Three main types of adhesive were used to glue the windows onto the cells: Superglue (a cyanoacrylate), Loctite 'Double
Bubble' (a 2-part epoxy adhesive) and Araldite 2010. Initial tests with the different adhesives showed that each one had varying performance with respect to sealing the gas cell windows and foils. Using a gas-fill rig (described in Section 4) the bonds were tested on a range of filled targets and it was found that the adhesives with the shortest cure times (Superglue and Double Bubble) in almost all cases failed at approximately 1 bar pressure with the windows lifting away from the aluminium body in places. In contrast, Araldite 2010, which has a 24-h cure to reach a lap shear strength of $10 \mathrm{MPa}^{[10]}$, exhibited excellent behaviour and was chosen to assemble the rest of the target components.

\section{Target filling and testing}

A system for filling targets with gas (Figure 4) was built by Imperial College, based on a similar system originally designed by the Lawrence Livermore National Laboratory and currently in use at the Laboratory for Laser Energetics ${ }^{[1]}$. The target was placed in a vacuum chamber (Figure 5) and the fill tube of the target connected to a separate line allowing it to be pumped and re-filled independently to the main chamber. This line was attached to a pressure transducer and the volume of the line and the transducer acted as a reservoir to maintain the pressure in the target for a prolonged period of time. Monitoring of pressure transducers allowed a leak test to be performed on the target and imaging of the target windows allowed for a deflection test to be conducted.

The deflection tests on the end windows (pusher) of the target were important as the Orion laser beams would be focused onto a specific point on the target and this point would be referenced from alignment marks and features. A gas target in the Orion target chamber that had a foil deflection and therefore offset from its intended position would mean a defocused beam on the surface and a lower intensity than expected. The results from the first deflection test in which a target was pressurized in an evacuated
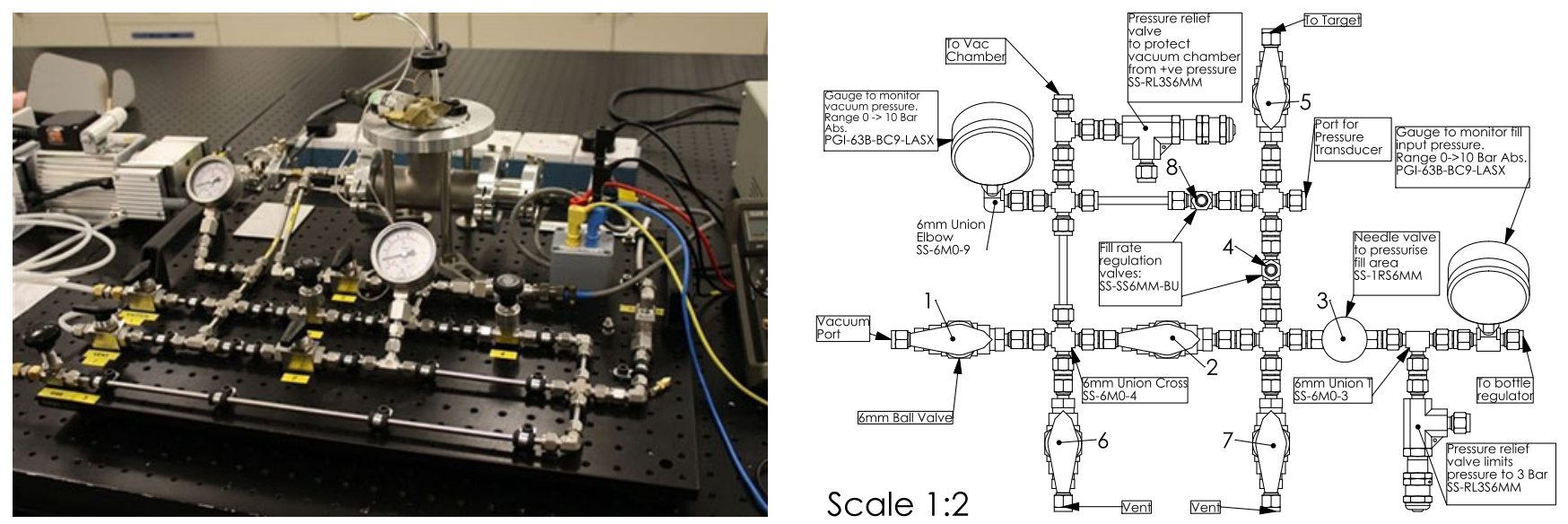

Figure 4. (a) Picture and (b) schematic of the gas system used for filling targets to specified pressures. 


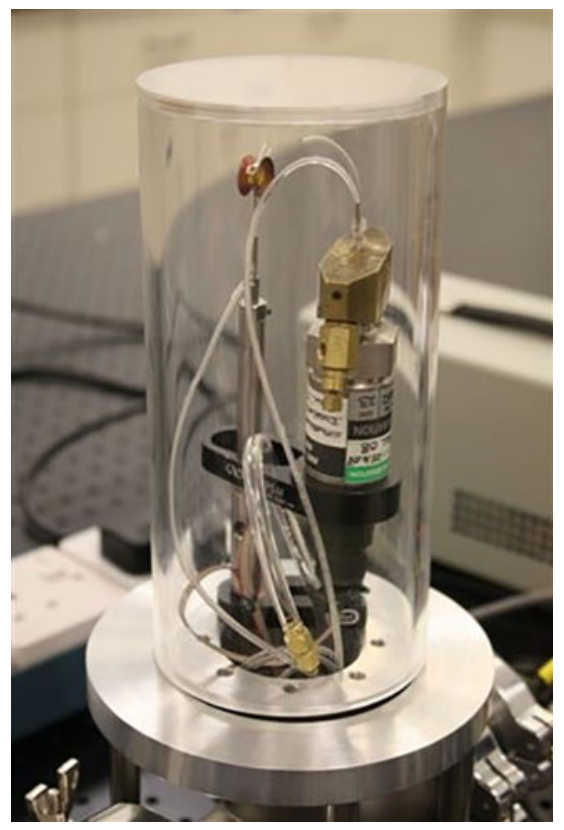

Figure 5. Target under vacuum in the testing chamber with the attached transducer.

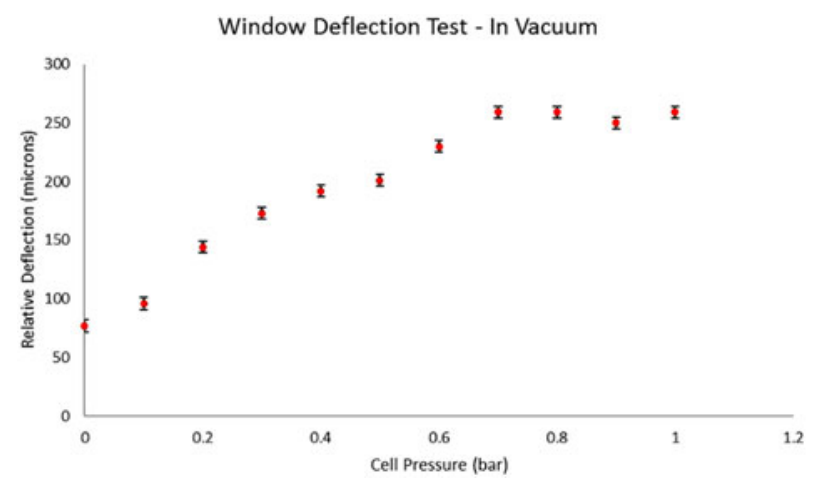

Figure 6. Deflection of a target in vacuum at a range of fill pressures.

testing chamber (see Figure 6) showed that there was bowing outwards of the pusher target end windows between 75 and $250 \mu \mathrm{m}$ depending on cell pressure. This measurement was taken from the gas cell body and therefore there is a $75 \mu \mathrm{m}$ initial offset due to the foil thicknesses. The deflection was reasonably consistent across the range of targets and was used to determine the offset of the laser focal spots to ensure the correct laser interaction plane.

A second set of tests was carried out using a target that was filled in the range of 0.1-1 bar in an external environment of atmospheric pressure. This test was carried out to test the cell integrity when transporting from the testing chamber to the Orion target chamber. In this case the target would be at a lower pressure than the external environment. The target bowed inwards and the relative deflections are shown in Figure 7. Again it can be seen from the data that the
Window Deflection Tests - Atmospheric

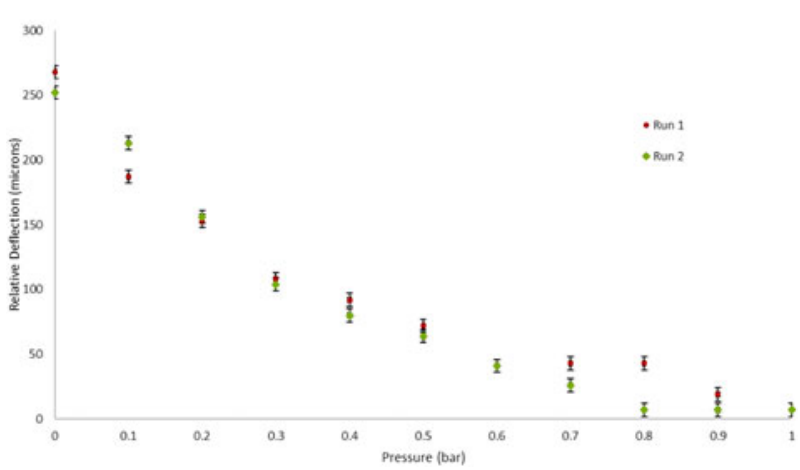

Figure 7. Deflection of target pusher window in an atmospheric pressure environment at a range of fill pressures.

deflection ranged from 75 to $250 \mu \mathrm{m}$ under a range of gas pressures and the integrity of the target under all conditions that would be encountered before the shot had also been confirmed.

Errors in both deflection tests are determined from the observed variation in the confocal positioning sensor that was used to measure the position of the foil. This is observed to vary when measuring a static foil by $\pm 5 \mu \mathrm{m}$ due to the vibrations inherent in the system that move the target. The variation in the pressure as measured by the transducer is again determined by observation of the transducer measurement on the gas-fill rig. This takes out any variations from the target itself and can be seen to be \pm 1 mbar. Transducers are provided calibrated from the supplier.

\section{Pressure testing and experimental performance}

Initial pressure testing showed an average leak rate of 5-10 mbar/min. This was significantly improved to $2 \mathrm{mbar} / \mathrm{min}$ by using (1) fixed attachments to the targets; (2) removable gas-tight fittings to transfer the targets from the fill station to the chamber; (3) a thorough and robust pressure test regime.

Three 1-week experimental campaigns were carried out in March 2015, July 2015 and October 2015. In addition to the gas cell targets backlighter targets used were a standard design as in previous campaigns ${ }^{[8]}$.

The leak rates for the targets when in the chamber were improved from the first to the second weeks of shots by the aforementioned steps and the target shot leak rates can be seen in Figure 8, with the sudden drops in pressure attributed to the shot breaking apart the cell.

The leak rates, improved from the first tests, were characterized to be approximately 1-2 mbar per minute in the first week targets with the improved fabrication and adhesive performance leading to the production of targets that could hold pressure at a relatively stable level for up to $2 \mathrm{~h}$ for the 


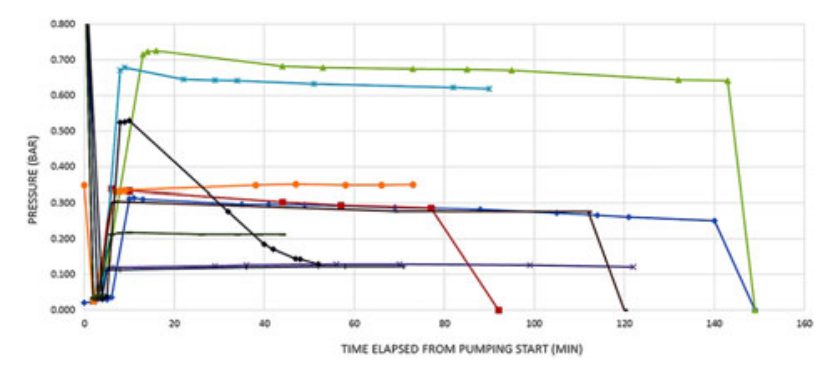

Figure 8. Target leak rates for a number of targets in the Orion chamber (sample time every $10 \mathrm{~min}$ ).

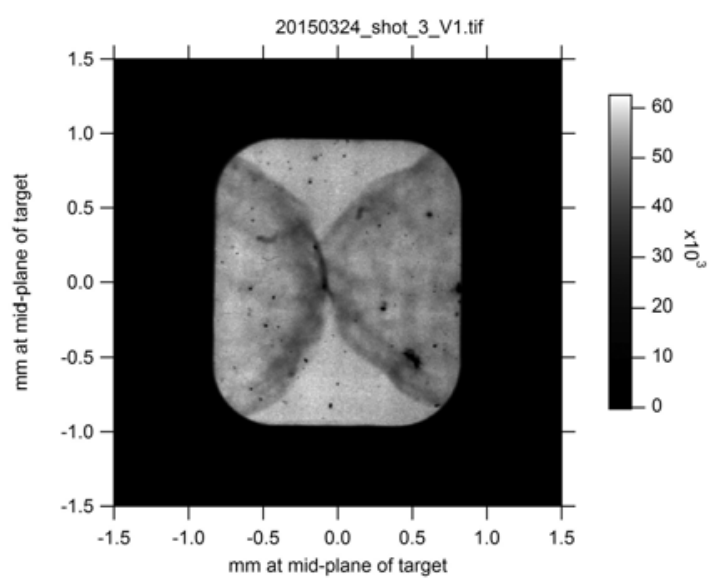

Figure 9. Example X-ray backlighting data from the Orion Imperial College academic access experiment.

subsequent experiment. This was essential as the target was installed in the Orion chamber and held at pressure and the shots were prepared. It can be seen in Figure 8, where the pressure in a number of targets is plotted over time, that the pressure is stable until the shot when the graph shows a rapid decrease in pressure as the cell is ruptured. It can also be seen that for some targets there was a significant leak after filling and this target did not meet specifications.

\section{X-ray target imaging}

A sample backlighter image is shown in Figure 9. The two shocks can be observed having propagated into the target and colliding in the middle of the gas cell. The data was taken by inserting in the chamber a backlighter target mounted onto a TIM to allow accurate positioning of the target to image through a set of $25 \mu \mathrm{m}$ thick polyimide windows on the cell and onto image plates. The backlighter target was a $5 \mu \mathrm{m}$ thick iron foil mounted over a $20 \mu \mathrm{m}$ diameter pinhole laser milled in $100 \mu \mathrm{m}$ thick tungsten foil, and this defined the resolution of the image ${ }^{[12]}$. This target was irradiated by two Orion backlighter beams.

\section{Conclusions}

A total of 164 targets (75 gas cells and 89 backlighters) were manufactured for three separate 1-week academic access experiments on Orion. The development of several new target fabrication techniques in collaboration with Imperial College London and AWE has enhanced the capabilities that are available and has contributed to a highly successful academic access campaign ${ }^{[2,3]}$. Further work is to be carried out to enhance the quality of the gas cell targets to enable further experiments with other geometries.

\section{References}

1. N. Hopps, C. Danson, S. Duffield, D. Egan, S. Elsmere, M. Girling, E. Harvey, D. Hillier, M. Norman, S. Parker, P. Treadwell, D. Winter, and T. Bett, Appl. Opt. 52, 3597 (2013).

2. T. Clayson, F. Suzuki-Vidal, S. V. Lebedev, G. F. Swadling, C. Stehle, G. C. Burdiak, J. Foster, J. Skidmore, P. Graham, E. Gumbrell, S. Patankar, C. Spindloe, U. Chaulagain, M. Kozlova, J. Larour, R. L. Singh, R. A. Smith, R. Rodriguez, J. M. Gil, G. Espinosa, P. Velarde, and C. Danson, High Energy Density Physics 23, 60 (2017).

3. F. Suzuki-Vidal, T. Clayson, G. F. Swadling, C. Stehlé, S. V. Lebedev, J. M. Foster, J. Skidmore, G. C. Burdiak, P. Graham, E. Gumbrell, S. Patankar, C. Spindloe, M. Kozlova, J. Larour, U. Chaulagain, R. L. Singh, R. Rodriguez, J. M. Gil, G. Espinosa, P. Velarde, and C. Danson, Phys. Rev. Lett. 119, 055001 (2017).

4. C. M. Krauland, R. P. Drake, C. C. Kuranz, R. Sweeney, M. Grosskopf, S. Klein, R. Gillespie, P. A. Keiter, B. Loupias, and E. Falize, Phys. Plasmas 20, 056502 (2013).

5. C. Stehle, M. Gonzalez, M. Kozlova, B. Rus, T. Mocek, O. Acef, J.-P. Colombier, T. Lanz, N. Champion, K. Jakubczak, J. Polan, P. Barroso, D Bauduin, E. Audit, J. Dostal, and M. Stupka, Laser Part. Beams 28, 253 (2010).

6. U. Chaulagain, C. Stehle, J. Larourc, M. Kozlova, F. SuzukiVidal, P. Barroso, M. Cotelo, P. Velarde, R. Rodriguez, J. M. Gil, A. Ciardi, O. Acef, J. Nejdl, L. de Sa, R. L. Singh, L. Ibgui, and N. Champion, High Energy Density Physics 17, 106 (2014).

7. A. Dizière, C. Michaut, M. Koenig, C. D. Gregory, A. Ravasio, Y. Sakawa, Y. Kuramitsu, T. Morita, T. Ide, H. Tanji, H. Takabe, P. Barroso, and J.-M. Boudenne, Astrophys. Space Sci. 336, 213 (2011).

8. C. Spindloe, D. Wyatt, D. Haddock, I. East, J. E. Cross, C. N. Danson, E. Falize, J. M. Foster, M. Koenig, and G. Gregori, High Power Laser Sci. Eng. 3, e8 (2015).

9. Analysis report from Loughborough Surface Analysis Ltd (Oct 2014).

10. Technical data sheet, http://www.intertronics.co.uk/data/ara20 10-1.pdf.

11. J. M. Foster, Internal AWE communication.

12. C. C. Kuranz, B. E. Blue, R. P. Drake, H. F. Robey, J. F. Hansen, J. P. Knauer, M. J. Grosskopf, C. Krauland, and D. C. Marion, Rev. Sci. Instrum. 77, 10E327 (2006). 